\title{
Analysis and Optimal Control in the Cancer Treatment Model by Combining Radio and Anti-angiogenic Therapy
}

\author{
Nastitie and Didik Khusnul Arif
}

\begin{abstract}
Cancer is a disease characterized by uncontrolled cell division and the ability of these cells to invade other tissues, either tissue next to them or not. Cancer treatment process is very diverse. The issue in this paper is about the treatment of cancer that processed by combining radio and anti-angiogenic therapy, which is intended to minimize the size of cancer, by adding control $w$ (dose of radiotherapy) and $u$ (dose of antiangiogenic). By applying Pontryagin minimum principle, the simulation results show that the size of the cancer become the most minimum if given minimum weight of anti-angiogenic $(P)$ therapy is 0.3 and radiotherapy $(Q)$ is 0.015 . Which take big effect of this treatment is dose of anti-angiogenic therapy.

Index Terms-Anti-angiogenic agent, Pontryagin minimum principle, radiotherapy.
\end{abstract}

\section{INTRODUCTION}

C ANCER is a disease characterized by uncontrolled cell division and ability of these cells to invade other tissues, either tissue next to them or not. Cancer is one of the disease that caused mortality in the world. More than $30 \%$ of cancer deaths are caused by five behavioral risk factors and diet that is high body mass index, less consumption of fruits and vegetables, lack of physical activity, tobacco, and excessive alcohol consumption. Estimated annual cancer cases will increase from 14 million in 2012 to 22 million in the next two decades [1].

The increasing number of cancer patients resulted in the medical world to find ways to prevent cancer or cancer treatment method. More than $30 \%$ of cancer can be prevented by changing behavioral risk factors and dietary causes of cancer. When the stage prevention is not successful then the treatment process can be reached. Cancer treatment can be done with surgery, chemotherapy, radiation, or a combination between treatments [1].

The main principle of radiotherapy which uses ionizing radiation to damage the genetic material of cancer cells (DNA), causing cell death or loss of ability to proliferation. However, radiotherapy side effects that exposure to radiation can damage normal tissue around the cancer cells. Realized that radiotherapy has low efficiency level and high number of toxicity to the cells of non-cancerous, doctors try to develop gene therapies that can improve the efficiency of the therapy,

Manuscript received May 22, 2017; accepted June 9, 2017.

The authors are with the Department of Mathematics, Institut Teknologi Sepuluh Nopember, Surabaya 60111, Indonesia. Email: didikematematika.its.ac.id while reducing the level of toxicity to the cells of noncancerous, by attacking the blood vessels of cancer known as anti-angiogenic therapy.

Anti-angiogenic therapy is usually given to patients that have last stages of cancer and radiotherapy can be applied at all levels of the cancer. When radiotherapy is combined with anti-angiogenic agent, radiotherapy will help reduce the pain suffered by patients. On the other hand, anti-angiogenic hold up the supply of nutrients and oxygen to the cancer cells. So with combination the two treatments is expected to shrink the size of the cancer and hold up the metastasis of cancer and the patient's life expectancy may increase.

Based on the description above, in this research, we will analyze dynamical model and establish an optimal control in the treatment model of cancer by combining radio and anti-angiogenic therapy. After optimal control is obtained, we will analyze the relevance or the effect of each variable on mathematical models.

\section{Research Methodology}

Mathematical models of cancer treatment by combining radio and anti-angiogenic therapy is a non-linear model, so to analyze the dynamic model, we must go through linearization process. After the system is linear, it can be analyzed whether the system is stable, controllable, observable or not.

The next step is to form optimal control using Pontryagin minimum principle, with the objective function of minimizing the size of cancer. Then, we calculate using numerical order Runge-Kutta method 4, and the results of the simulation analysis on the relevance of each variable.

\section{Results AND Discussions}

\section{A. Analysis of Dynamical Model}

The mathematical models used in this problem are [2]:

$$
\begin{aligned}
\dot{p} & =-\xi p \ln \frac{p}{q}-(\alpha+\beta r) p w \\
\dot{q} & =b p-\left(\mu+d p^{\frac{2}{3}}\right) q-\gamma q u-(\eta+\delta r) q w \\
\dot{r} & =-\rho r+w \\
\dot{y} & =u \\
\dot{z} & =(1+\theta s) w \\
\dot{s} & =-\sigma s+w
\end{aligned}
$$

where: 
- $p$ primary tumor volume

- $q$ carrying capacity of the tumor vasculature

- $r$ tissue repair

- $y$ accumulated amount of used anti-angiogenic agent

- $z$ accumulated radiation dose in tumor

- $s$ accumulated radiation dose in healty tissue

- $u$ dosage of anti-angiogenic agent

- $w$ radiation dose

- $\alpha, \eta$ linear damage caused by radiation of the tumor

- $\beta, \delta$ quadratic damage caused by radiation of the tumor

- $\rho$ repair rate of the tumor and endothelial cells

- $\sigma$ repair rate for the healty cells

- $\theta$ parameter of the healty tissue

- $b$ tumor-induces stimulation parameter

- $d$ tumor-induces inhibition parameter

- $\gamma$ anti-angiogenic elimination parameter

- $\xi$ tumor growth parameter

- $\mu$ baseline loss of vascular support through natural causes

1) Stability Analysis of the System: The mathematical model shown in equation (1)-(6) is non-linear, so the analysis of the stability to be around the point of equilibrium.

Definition 1 (Equilibrium Point [3]): Point $\bar{x} \in \mathbb{R}^{n}$ is called the equilibrium point of the system if $f(\bar{x})=0$.

From Definition 1, we obtained the following 3 equilibrium points:

$$
\begin{aligned}
& E_{1}=\left(\left(\frac{b-\mu}{d}\right)^{\frac{3}{2}},\left(\frac{b-\mu}{d}\right)^{\frac{3}{2}}, 0, c_{1}, c_{2}, 0\right) \\
& E_{2}=\left(\frac{\left(-\left(\eta-\delta \frac{\sigma}{\theta \rho}\right) \frac{\sigma}{\theta}+b e^{\frac{\left(\alpha-\beta \frac{\sigma}{\theta \rho}\right) \frac{\sigma}{\theta}}{\xi}}-\mu\right)^{\frac{3}{2}}}{d^{\frac{3}{2}}},\right. \\
& \frac{\left(-\left(\eta-\delta \frac{\sigma}{\theta \rho}\right) \frac{\sigma}{\theta}+b e^{\frac{\left(\alpha-\beta \frac{\sigma}{\theta \rho}\right) \frac{\sigma}{\theta}}{\xi}}-\mu\right)^{\frac{3}{2}}}{d^{\frac{3}{2}} e^{\frac{\left(\alpha-\beta \frac{\sigma}{\theta \rho}\right) \frac{\sigma}{\theta}}{\xi}}},-\frac{\sigma}{\theta \rho}, c_{1}, c_{2},-\frac{1}{\theta}
\end{aligned}
$$$$
E_{3}=\left(-\left(\eta-\delta \frac{\sigma}{\theta \rho}\right) \frac{\sigma}{\theta} e^{\frac{\left(\alpha-\beta \frac{\sigma}{\theta \rho}\right) \frac{\sigma}{\theta}}{\xi}},-\left(\eta-\delta \frac{\sigma}{\theta \rho}\right) \frac{\sigma}{\theta},-\frac{\sigma}{\theta \rho}\right.
$$$$
\left.c_{1}, c_{2},-\frac{1}{\theta}\right)
$$

Then, Taylor series are used to analyze the stability of the system around the equilibrium point and in the form of Jacobian. The Jacobian matrix of this mathematical model is:

$$
\left(\begin{array}{c}
\dot{p} \\
\dot{q} \\
\dot{r} \\
\dot{y} \\
\dot{z} \\
\dot{s}
\end{array}\right)=\left(\begin{array}{cccccc}
A_{11} & A_{12} & A_{13} & 0 & 0 & 0 \\
A_{21} & A_{22} & A_{23} & 0 & 0 & 0 \\
0 & 0 & A_{33} & 0 & 0 & 0 \\
0 & 0 & 0 & 0 & 0 & 0 \\
0 & 0 & 0 & 0 & 0 & A_{56} \\
0 & 0 & 0 & 0 & 0 & A_{66}
\end{array}\right)\left(\begin{array}{c}
\hat{p} \\
\hat{q} \\
\hat{r} \\
\hat{y} \\
\hat{z} \\
\hat{s}
\end{array}\right)
$$

where:

$$
\begin{aligned}
& A_{11}=-\xi\left(\ln \frac{p}{q}+1\right)-(\alpha+\beta r) w \\
& A_{12}=\frac{\xi p}{q} \\
& A_{13}=-\beta p w \\
& A_{21}=b-\frac{2}{3} d q p^{-\frac{1}{3}} \\
& A_{22}=-\left(\mu+d p^{\frac{2}{3}}\right)-\gamma u-(\eta+\delta r) w \\
& A_{23}=-\delta q w \\
& A_{33}=-\rho \\
& A_{56}=\theta w \\
& A_{66}=-\sigma
\end{aligned}
$$

Theorem 2 ([4]): Given a differential equation $\dot{x}=A x$ where $A$ is a matrix of size $n \times n$ and have different characteristic values $\lambda_{1}, \ldots, \lambda_{k}(k \leq n)$. The origin point $x=0$ is asymptotically stable if and only if the real parts of $\lambda_{i}<0$ for $i=1, \ldots, k$. The point of origin is stable if the real value of $\lambda_{i} \leq 0$ with $i=1, \ldots, k$.

From Theorem 2, we can determine the system's stability around the equilibrium points $E_{1}, E_{2}, E_{3}$. For $E_{1}$, the system is stable, whereas for $E_{2}$ and $E_{3}$ the system is unstable.

2) Controllability Analysis: In this subsection, we describe a method to check whether a system is controllable or not.

Theorem 3 ([3]): Given the following LTI system:

$$
\left.\begin{array}{l}
\dot{x}(t)=A x(t)+B u(t) \\
y(t)=C x(t)
\end{array}\right\}
$$

A necessary and sufficient condition for LTI system (7) to be controllable is that the controllability matrix $M_{c}=$ $\left(B|A B| A^{2} B|\ldots| A^{n-1} B\right)$ has rank equal to $n$.

After we compute $M_{c}$ by using the above formula, we determine the rank of $M_{c}$. It turns out that the rank of $M_{c}$ is 6 , thus the system is controllable.

3) Observability Analysis: In this subsection, we describe a method to check whether a system is observable or not.

Theorem 4 ([5]): In (7), a necessary and sufficient condition of a system to be observable is that the following observability matrix

$$
M_{o}=\left[\begin{array}{c}
C \\
C A \\
C A^{2} \\
\vdots \\
C A^{(n-1)}
\end{array}\right]
$$

has rank equal to $n$.

After determining $M_{o}$, we found that rank of $M_{o}$ is not equal to 6. From Theorem 4, the system is unobservable.

\section{B. Formulation of the Optimal Control}

1) Formulation of the Objective Function: Goal of this research is minimizing the size of cancer $(p)$ by combining radio $(w)$ and anti-angiogenic $(u)$ therapy. so that the objective function can be formulated as follows:

$$
J(w, u)=\int_{t_{0}}^{t_{f}}\left(p+\frac{Q}{2} u(t)^{2}+\frac{P}{2} w(t)^{2}\right) d t
$$


2) Formulation of the Hamilton Function:

$$
\begin{aligned}
& H(p, q, r, y, z, s, w, u, \lambda)=\left(p+\frac{Q}{2} u(t)^{2}+\frac{P}{2} w(t)^{2}\right)+\sum_{i=1}^{6} \lambda_{i} f_{i} \\
& H=\left(p+\frac{Q}{2} u(t)^{2}+\frac{P}{2} w(t)^{2}\right)+\lambda_{1}\left(-\xi p \ln \frac{p}{q}-(\alpha+\beta r) p w\right) \\
& +\lambda_{2}\left(b p-\left(\mu+d p^{\frac{2}{3}}\right) q-\gamma q u-(\eta+\delta r) q w\right)+ \\
& \lambda_{3}(-\rho r+w)+\lambda_{4}(u)+\lambda_{5}((1+\theta s) w)+\lambda_{6}(-\sigma s+w) \\
& \frac{\partial H}{\partial w}=P w(t)-\lambda_{1}(\alpha+\beta r) p-\lambda_{2}(\eta+\delta r) q+\lambda_{3}+ \\
& \lambda_{5}(1+\theta s)+\lambda_{6}=0 \\
& w(t)=\frac{\lambda_{1}(\alpha+\beta r) p+\lambda_{2}(\eta+\delta r) q-\lambda_{3}-\lambda_{5}(1+\theta s)-\lambda_{6}}{P}
\end{aligned}
$$

So we obtain the radiotherapy control limit values, which are given by

$$
\begin{gathered}
w:[0, T] \rightarrow \min \{1, \max [w(t)]\} . \\
\frac{\partial H}{\partial u}=Q u(t)-\lambda_{2}(\gamma q)+\lambda_{4}=0 \\
u(t)=\frac{\lambda_{2}(\gamma q)-\lambda_{4}}{Q}
\end{gathered}
$$

And the limit value of anti-angiogenic therapy is $u:[0, T] \rightarrow$ $\min \{1, \max [u(t)]\}$

4) Determine $H^{*}$ :

$$
\begin{aligned}
H^{*} & =\left(p+\frac{Q}{2} u(t)^{2}+\frac{P}{2} w(t)^{2}\right)+ \\
& \lambda_{1}\left(-\xi p \ln \frac{p}{q}-(\alpha+\beta r) p \min \{1, \max [0, w(t)]\}\right)+ \\
& \lambda_{2}\left(b p-\left(\mu+d p^{\frac{2}{3}}\right) q-\gamma q \min \{1, \max [0, u(t)]\}-\right. \\
& (\eta+\delta r) q \min \{1, \max [0, w(t)]\})+ \\
& \lambda_{3}(-\rho r+\min \{1, \max [0, w(t)]\})+ \\
& \lambda_{4}(\min \{1, \max [0, u(t)]\})+ \\
& \lambda_{5}((1+\theta s) \min \{1, \max [0, w(t)]\})+ \\
& \lambda_{6}(-\sigma s+\min \{1, \max [0, w(t)]\})
\end{aligned}
$$

5) Determine state functions:

$$
\begin{aligned}
& \frac{\partial H^{*}}{\partial \lambda_{1}}=-\xi p \ln \frac{p}{q}-(\alpha+\beta r) p \min \{1, \max [0, w(t)]\} \\
& \frac{\partial H^{*}}{\partial \lambda_{2}}=b p-\left(\mu+d p^{\frac{2}{3}}\right) q-\gamma q \min \{1, \max [0, u(t)]\}- \\
& \quad(\eta+\delta r) q \min \{1, \max [0, w(t)]\} \\
& \frac{\partial H^{*}}{\partial \lambda_{3}}=-\rho r+\min \{1, \max [0, w(t)]\} \\
& \frac{\partial H^{*}}{\partial \lambda_{4}}=\min \{1, \max [0, u(t)]\} \\
& \frac{\partial H^{*}}{\partial \lambda_{5}}=(1+\theta s) \min \{1, \max [0, w(t)]\} \\
& \frac{\partial H^{*}}{\partial \lambda_{6}}=-\sigma s+\min \{1, \max [0, w(t)]\}
\end{aligned}
$$

6) Determine costate functions:

$$
\begin{aligned}
& \frac{\partial \lambda_{1}}{\partial t}=-\frac{\partial H^{*}}{\partial p}=-\left(1+\lambda_{1}\left(-\xi\left(\ln \frac{p}{q}+1\right)-\right.\right. \\
&\left.(\alpha+\beta r) \min \{1, \max [0, w(t)]\})+\lambda_{2}\left(b-\frac{2}{3} d q p^{-\frac{1}{3}}\right)\right) \\
& \frac{\partial \lambda_{2}}{\partial t}=-\frac{\partial H^{*}}{\partial q}=-\left(\lambda_{1}\left(\frac{\xi p}{q}\right)+\lambda_{2}\left(-\left(\mu+d p^{\frac{2}{3}}\right)-\right.\right. \\
&\gamma \min \{1, \max [0, u(t)]\}-(\eta+\delta r) \min \{1, \max [0, w(t)]\})) \\
& \frac{\partial \lambda_{3}}{\partial t}=-\frac{\partial H^{*}}{\partial r}=-\left(\lambda_{1}(-\beta \min \{1, \max [0, w(t)]\})+\right. \\
&\left.\lambda_{2}(-\delta q \min \{1, \max [0, w(t)]\})+\lambda_{3}(-\rho)\right) \frac{\partial \lambda_{4}}{\partial t}=-\frac{\partial H^{*}}{\partial y}=0 \\
& \frac{\partial \lambda_{5}}{\partial t}=-\frac{\partial H^{*}}{\partial z}=0 \\
& \frac{\partial \lambda_{6}}{\partial t}=-\frac{\partial H^{*}}{\partial s}=-\left(\lambda_{5}(\theta \min \{1, \max [0, w(t)]\})+\lambda_{6}(-\sigma)\right)
\end{aligned}
$$

In the next step, the result from point $1-6$ is solved numerically by using fourth-order Runge Kutta.

\section{Simulation Results}

In the simulation, we use the same initial condition $(0.9 ; 0.55 ; 0.3 ; 0.1 ; 0.01 ; 0.1)$ and we give a different dosage of anti-angiogenic and radiotherapy. The following results are obtained :

1) Dosage of radiotherapy is greater than anti-angiogenic there is $0.6 \mathrm{~Gy}$ for radiotherapy and $0.3 \mathrm{mg} / \mathrm{kg}$ for anti-angiogenic obtained objective function value is $0.1814 \mathrm{~mm}^{3}$.

2) Dosage of radiotherapy is smaller than anti-angiogenic there is $0.3 \mathrm{~Gy}$ of radiotherapy and $0.6 \mathrm{mg} / \mathrm{kg}$ for anti-angiogenic obtained objective function value is $0.1813 \mathrm{~mm}^{3}$.

3) Dosage of radiotherapy as much as anti-angiogenic dosage there is $0.3 \mathrm{~Gy}$ of radiotherapy and $0.3 \mathrm{mg} / \mathrm{kg}$ for anti-angiogenic obtained objective function value of $0.1814 \mathrm{~mm}^{3}$.

From threee experiments above, dosage of anti-angiogenic which is more than radiotherapy proved to be more effective to suppress the development of cancer. The following chart shows figures of radiotherapy and anti-angiogenic dosages that are most effective:

Figures 1 and 2 above show that the dosage of antiangiogenic is greater than radiotherapy. But anti-angiogenic dosage is decreased over time. It is offset by the provision of radiotherapy which tends to a constant every day. The treatment process as shown above has proven effective to inhibit the growth of cancer.

Then we do the experiment with the dosage of antiangiogenic $0.6 \mathrm{mg} / \mathrm{kg}$ and radiation $0.3 \mathrm{~Gy}$ at different initial conditions by noting that the size of the blood vessels of cancer is smaller than an area of normal tissue exposed by radiation, the size of the vessel blood cancer is greater than an area of normal tissue exposed by radiation, and the size of the 


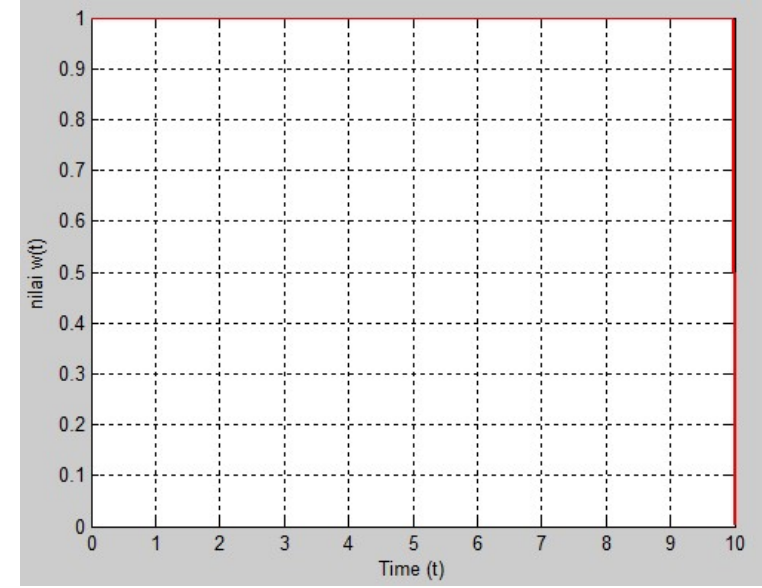

Fig. 1. Dosage of radiotherapy

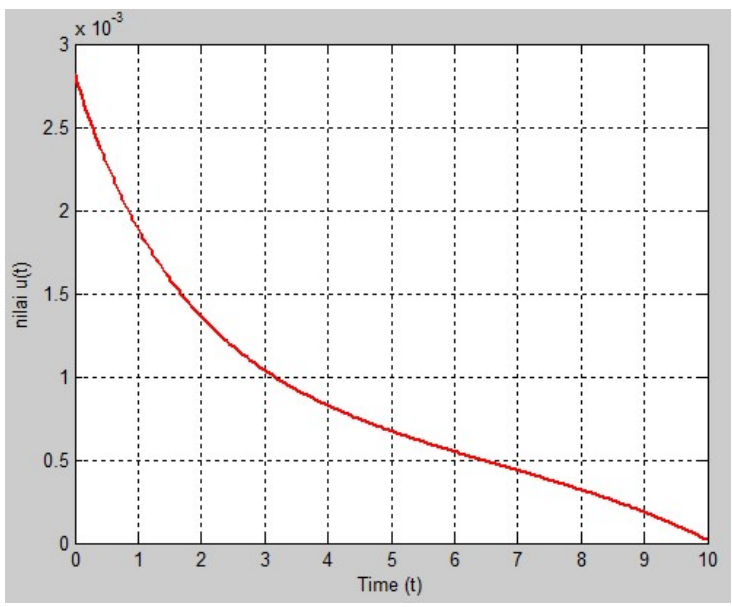

Fig. 2. Dosage of anti-angiogenic agent

blood vessels as much as an area of normal tissue exposed by radiation.

1) The initial conditions $(0.9 ; 0.3 ; 0.3 ; 0.1 ; 0.01 ; 0.7)$ indicate that the size of blood vessels is smaller than an area of normal tissue exposed by radiation. Based on initial conditions found that anti-angiogenic's ability to attack the blood vessels of cancer is 3, the ability of radiotherapy to attack non-cancerous tissue is 0.0014 and the ability of non-cancerous tissue that is damaged to repair themselves is $2: 33$.

2) The initial conditions $(0.9 ; 0.6 ; 0.3 ; 0.1 ; 0.01 ; 0.6)$ indicate that the size of the blood vessels of cancer as much as an area of normal tissue exposed by radiation, showed the ability of radiotherapy to damage normal tissue surrounding the cancer cells is 2 and the network's ability to renew damaged is 0.0167 . While anti-angiogenic ability to suppress the growth of cancer blood vessels is 6 .

By comparing the above experimental results, we concluded that a dosage of anti-angiogenic agent is $0.0031 \frac{\mathrm{mg}}{\mathrm{kg}}$ and $0.9997 \mathrm{~Gy}$ for radiotherapy suitable to be applied in the initial conditions $(0.9 ; 0.3 ; 0.3 ; 0.1 ; 0.01 ; 0.7)$. The following chart will be displayed for each variable in the initial conditions with dosage of radiotherapy and anti-angiogenic has been determined:

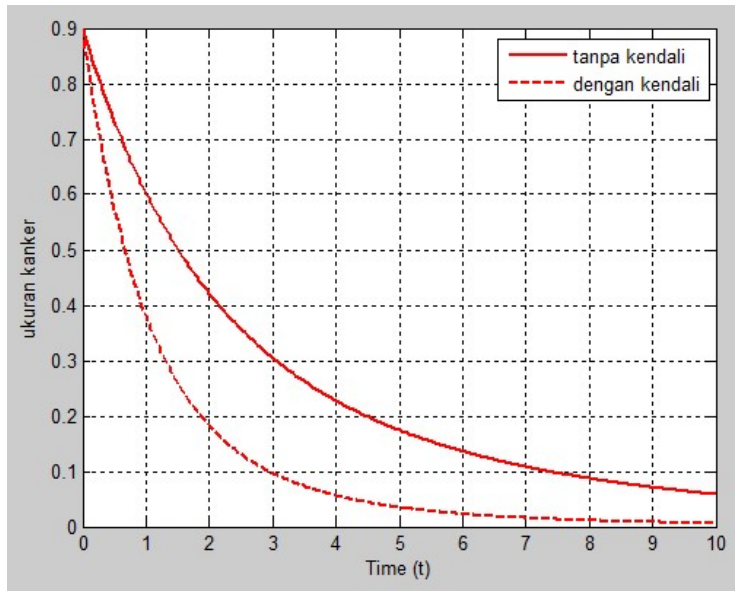

Fig. 3. Size of cancer

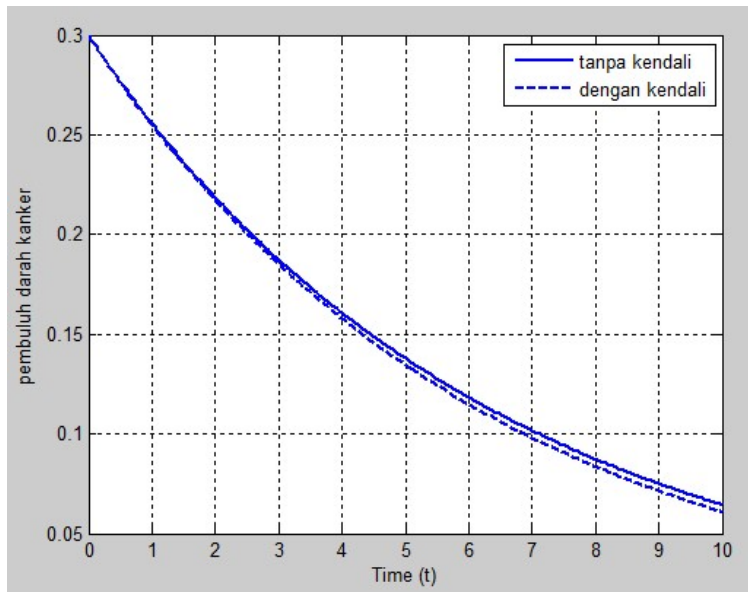

Fig. 4. Size of cancer's blood vessel

Figure 3 - Figure 8 show the changes of the variable given at different initial condition, but given the same dosage as in Experiment 2. The size of the initial cancer blood vessels of $0.3 \mathrm{~mm}^{3}$ by giving anti-angiogenic of $0.0031 \mathrm{mg} / \mathrm{kg}$ and normal tissue exposed to radiation of 0.7 by giving radiotherapy of $0.9997 \mathrm{~Gy}$, the size of the cancer at day 10 is $0.0062 \mathrm{~mm}^{3}$ was followed by a decrease in the size of the blood vessel to a size of $0.0608 \mathrm{~mm}^{3}$ with the body's absorption of the anti-angiogenic at $0.0075 \mathrm{mg}$.

Thus, it can be concluded that treatment with combined radiotherapy and anti-angiogenic depends on the size of the blood vessels of cancer formed by cancerous and focus whether irradiation radiotherapy that is characterized by the extent of normal tissue exposed to radiation.

\section{Conclusions}

1) In the model of treatment of cancer by combining radiotherapy and anti-angiogenesis, we concluded that the system is controlled at the point of equilibrium:

$E_{1}=\left(\left(\frac{b-\mu}{d}\right)^{\frac{3}{2}},\left(\frac{b-\mu}{d}\right)^{\frac{3}{2}}, 0, c_{1}, c_{2}, 0\right)$ 


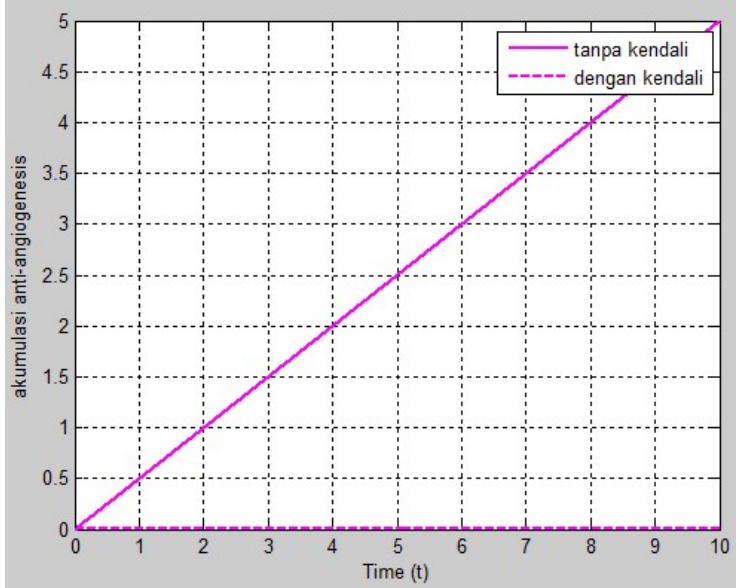

Fig. 5. Accumulation of anti-angiogenic agent

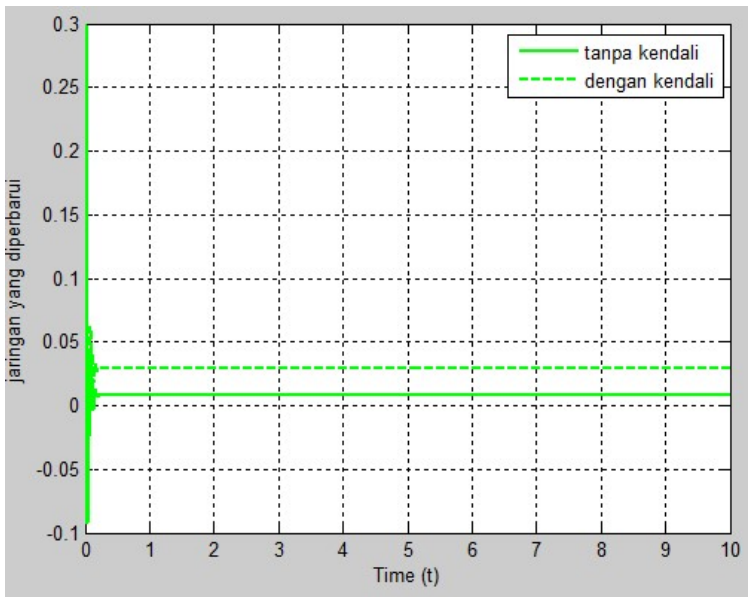

Fig. 6. Tissue repair

$$
\begin{aligned}
E_{2}= & \left(\frac{\left(-\left(\eta-\delta \frac{\sigma}{\theta \rho}\right) \frac{\sigma}{\theta}+b e^{\frac{\left(\alpha-\beta \frac{\sigma}{\theta \rho}\right) \frac{\sigma}{\theta}}{\xi}}-\mu\right)^{\frac{3}{2}}}{d^{\frac{3}{2}}},\right. \\
& \frac{\left(-\left(\eta-\delta \frac{\sigma}{\theta \rho}\right) \frac{\sigma}{\theta}+b e^{\frac{\left(\alpha-\beta \frac{\sigma}{\theta \rho}\right) \frac{\sigma}{\theta}}{\xi}}-\mu\right)^{\frac{3}{2}}}{d^{\frac{3}{2}} e^{\frac{\left(\alpha-\beta \frac{\sigma}{\theta \rho}\right) \frac{\sigma}{\theta}}{\xi}},} \\
& \left.-\frac{\sigma}{\theta \rho}, c_{1}, c_{2},-\frac{1}{\theta}\right) \\
E_{3} & =\left(-\left(\eta-\delta \frac{\sigma}{\theta \rho}\right) \frac{\sigma}{\theta} e^{\frac{\left(\alpha-\beta \frac{\sigma}{\theta \rho}\right) \frac{\sigma}{\theta}}{\xi}},-\left(\eta-\delta \frac{\sigma}{\theta \rho}\right) \frac{\sigma}{\theta},\right. \\
& \left.-\frac{\sigma}{\theta \rho}, c_{1}, c_{2},-\frac{1}{\theta}\right)
\end{aligned}
$$

The system is only stable at $E_{1}$, while at the point $E_{2}$, $E_{3}$ are unstable. However, $E_{1}, E_{2}, E_{3}$ are controllable but not observable. Despite this, on model of the cancer

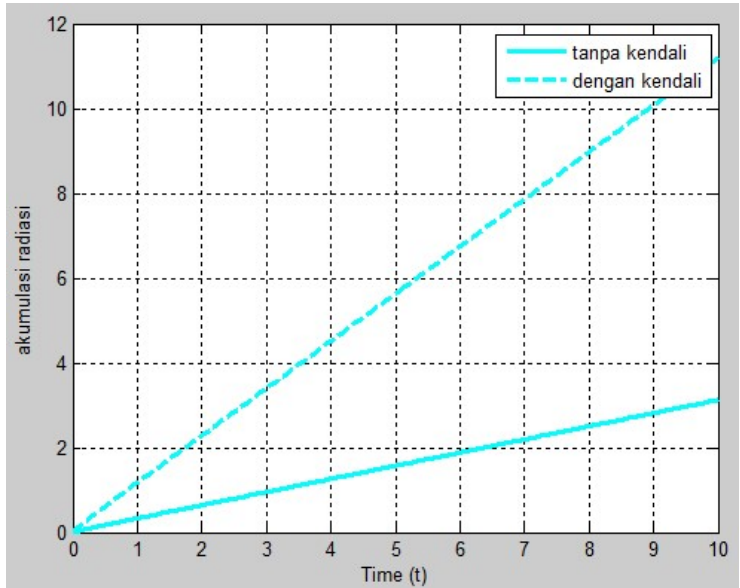

Fig. 7. Accumulation of radiotherapy

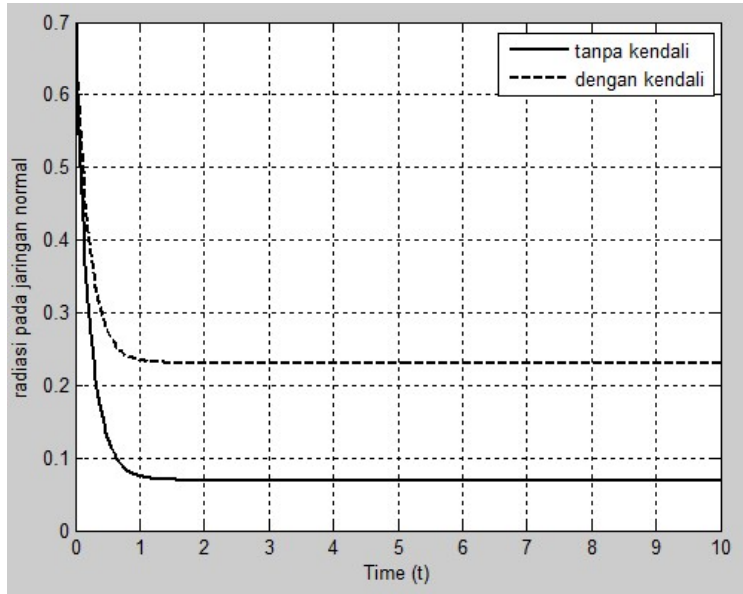

Fig. 8. Accumulation of radiation dosage in healty tissue

treatment we can formulate an optimal control problem. Thus, the main condition for formulating an optimal control system is the controllability of the system. If a system is not stable but the system is controllable, the system will be stable, and when the system is stable and controllable, the system will be accelerated to achieve stability.

2) The most optimal dosage of anti-angiogenic agent and radiotherapy given when the dosage of anti-angiogenic is greater than radiotherapy, which amounted to $0.0031 \mathrm{mg}$ / kg for anti-angiogenic and $0.9997 \mathrm{~Gy}$ for radiotherapy. Although, the dosage of anti-angiogenic quite a bit of administration but it is very effective to suppress the development of cancer.

3) From six variables on mathematical model there are the various sizes of the cancer, blood vessels of cancer, tissue repair, accumulation of anti-angiogenic, accumulation of radiotherapy, and accumulation of radiation dosage in healty tissue, the most influence to development cancer is blood vessels of cancer. Because blood vessels of cancer is formed simultaneously with the emergence of cancer in a network. The sooner action is taken, the blood vessels of cancer that forms in size 
is big enough and can still be overcomed.

\section{REFERENCES}

[1] Unknown, "Infodatin," Pusat Data dan Informasi Kementerian Kesehatan RI, Jakarta, Tech. Rep., 2010.

[2] K. Chudej, L. Wagner, and H. Pesch, "Numerical solution of an optimal control problem in cancer treatment: Combined radio and anti-angiogenic therapy," IFAC-PapersOnLine, vol. 48, no. 1, pp. 665-666, 2015.

[3] L. Perko, Differential equations and dynamical systems. Springer Science \& Business Media, 2013, vol. 7.

[4] Subiono, Matematika Sistem. Surabaya: Institut Teknologi Sepuluh Nopember, 2003.

[5] - Sistem Linear. Surabaya: Institut Teknologi Sepuluh Nopember, 2013. 